\title{
Dynamic Change of Fecal Calprotectin in Very Low Birth Weight Infants during the First Month of Life
}

\author{
Qing Yang ${ }^{\mathrm{a}, \mathrm{b}}$ P. Brian Smith ${ }^{\mathrm{a}}$ Ronald N. Goldberg ${ }^{\mathrm{a}}$ C. Michael Cotten ${ }^{\mathrm{a}}$ \\ a Division of Neonatology, Department of Pediatrics, Duke University Medical Center, Durham, N.C., and \\ ${ }^{b}$ Division of Neonatology, Department of Pediatrics, Wake Forest University Health Sciences, Winston-Salem, N.C., USA
}

\section{Key Words}

Fecal calprotectin - Very low birth weight infant •

Necrotizing enterocolitis $\cdot$ Diagnostic marker

\begin{abstract}
Background: Calprotectin is a cytosolic component of neutrophils. Fecal calprotectin (FC) level is a useful marker for exacerbation of inflammatory bowel disease in children. FC may be a useful marker for necrotizing enterocolitis (NEC). Objective: To determine normal baseline levels of FC and observe dynamic changes of FC levels over the first postnatal month in very low birth weight (VLBW) infants. Methods: FC levels of 14 VLBW infants (gestational age 23-30 weeks, birth weight $\leq 1,500 \mathrm{~g}$ ) were serially measured in the first postnatal month. Demographics, feeding regimens, antibiotic use, laboratory and $\mathrm{x}$-ray results, and maternal information were recorded. We assessed how $\mathrm{FC}$ levels changed over time, varied with nutritional source and differed between sick versus well infants. Results: FC levels were not related to gestational age or feedings regimen. FC levels tended to decrease with increasing age $(p=0.121)$ and feeding volumes $(p=0.179)$. FC levels differed between 'well' and 'sick' infants (122.8 \pm 98.9 vs. $380.4 \pm 246.3 \mu \mathrm{g} / \mathrm{g}$ stool, $\mathrm{p}<0.001)$. FC $>350 \mu \mathrm{g} / \mathrm{g}$ stool was noted with signs of gastrointestinal injury, such as bloody stool and bowel perforation. FC levels decreased after initiation of treatments in sick infants who
\end{abstract}

recovered. Conclusions: FC levels may be a marker for early diagnosis and resolution of gastrointestinal illnesses in VLBW infants. Its utility for early diagnosis and assessment of resolution of NEC should be studied in a larger cohort of VLBW infants.

Copyright $\odot 2008$ S. Karger AG, Basel

\section{Introduction}

Necrotizing enterocolitis (NEC) is the leading cause of mortality and morbidity from gastrointestinal disease in preterm neonates [1], and often occurs in the first 4 postnatal weeks [2]. The high incidence of NEC in premature infants with very low birth weight (VLBW; birth weight $<1,500 \mathrm{~g}$ ) [3], the lack of a specific clinical presentation, and the lack of a reliable biomarker underscores the need for a reliable, valid and accurate test for early diagnosis, which could reduce the morbidity and mortality of infants affected by NEC. A specific and reliable marker could provide early diagnosis and intervention for infants with true NEC, prevent other infants without NEC from receiving unnecessary treatment and prolonged cessation of enteral feedings, and provide a marker to monitor the response to NEC treatment.

Calprotectin, a calcium- and zinc-binding protein, constitutes approximately $60 \%$ of the cytosolic protein of

\section{KARGER}

Fax +41613061234 E-Mail karger@karger.ch www.karger.com (c) 2008 S. Karger AG, Basel

1661-7800/08/0944-0267\$24.50/0

Accessible online at:

www.karger.com/neo
Qing Yang, $\mathrm{MD}, \mathrm{PhD}$

Division of Neonatology, Department of Pediatrics

Wake Forest University Health Sciences

Medical Center Boulevard, Winston-Salem, NC 27157 (USA)

Tel. +1 336716 8687, Fax +1 336716 2525, E-Mail qyang@wfubmc.edu 
neutrophils, monocytes and macrophages [4]. Fecal calprotectin (FC) concentration positively and strongly correlates with fecal excretion of ${ }^{111}$ In-labeled granulocytes [5] and inflammatory bowel disease activity determined by histological and endoscopic evaluation [6]. FC levels have been used as an accurate marker of inflammatory bowel disease in both adults and children to assess disease activity $[7,8]$, but the diagnostic accuracy is higher in children [7]. In contrast, FC levels are not increased in infants with infantile colic or functional abdominal pain [8]. An age-dependent variation in FC levels has been found in children [9], with higher FC levels noted in newborns including healthy full-term [8-10], preterm [11] and VLBW infants [12]. FC was found to present in the first passed meconium in neonates [13] and increased in infants with NEC $[12,14]$.

Existing studies of FC in neonates are based on a single stool sample $[10,11,13,14]$, which did not account for the clinical condition of the infant, and there are sparse data regarding the normal range for $\mathrm{FC}$ levels in preterm infants. In addition, it is not known how FC levels change in preterm infants during the first month of life when most cases of NEC present [2]. Also unknown is whether FC levels change in response to changes in an infant's clinical status, as occurs with systemic infection, feeding intolerance, NEC, and treatment for NEC. Because FC level is elevated in old children with inflammation of the bowel, we hypothesized that FC levels might be a useful marker of the severity of gastrointestinal illnesses, such as NEC, and the response to treatment of such illnesses in VLBW infants. In this pilot study we serially measured FC levels daily or every other day under various clinical conditions from 14 VLBW infants to identify a normal baseline level of FC and to observe the dynamic change of FC levels over the first month of postnatal life. If the study results support FC's association with gastrointestinal illnesses and recovery, FC should be further assessed as a possible tool to diagnose NEC and assess recovery of NEC.

\section{Materials and Methods}

\section{Patients}

This prospective pilot study was approved by the Duke University Medical Center Institutional Review Board and carried out from February to May 2006. The study enrolled 14 VLBW infants consecutively born in Duke University Medical Center and admitted to Duke Neonatal Intensive Care Nursery after obtaining informed consent from the parent. Infants with congenital anomalies and those who were moribund were excluded from this study. Demographics, Apgar scores, daily feeding regimen, respiratory support, antibiotic use, laboratory and x-ray results, and maternal information for enrolled infants were recorded. Infants were classified as 'sick' if they underwent an evaluation for sepsis, required treatment with antibiotics or vasopressors, had feedings withheld, or required increased respiratory support.

\section{Sample Collection and FC Assay}

All stool samples were collected from enrolled infants during the first month of life. The data and time of each collection were recorded. All stool samples were stored at $-20^{\circ} \mathrm{C}$ before analysis. According to the availability of stool samples collected, the FC level was measured every other day or at least twice each week to determine the baseline level of FC during the infants' not sick period; otherwise in infants treated for suspected or proven infect, or exhibiting signs of gastrointestinal illness, FC levels were measured daily or from every consecutive stool sample to assess the dynamic change of the FC level during the infants' 'sick' and recovery period. FC levels were measured using PhiCal Test ELISA kit (Eurospital, Trieste, Italy; distributed by Genova Diagnostics Inc., Ashville, N.C., USA) following the manufacturer's instructions. In brief, about $40-60 \mathrm{mg}$ feces was used to extract $\mathrm{FC}$ from each stool sample. Duplicate supernatants containing FC were assayed. Results are reported as mean \pm SD in $\mu \mathrm{g} / \mathrm{g}$ stool.

\section{Statistical Analysis}

We used univariate analyses to determine the effect of gestational age (GA) at birth, day of life, feeding volume ( $\mathrm{ml} / \mathrm{kg} /$ day), type of feeding (maternal breast milk vs. hydrolyzed formula), and the clinical condition of the infant (sick vs. not sick) on FC levels. We incorporated GA at birth, day of life, feeding volume, and the clinical condition of the infants in a multivariate model to predict the FC level. Because there were several extremely high FC levels, the FC levels were transformed by taking the natural logarithm of FC levels for use in the uni- and multivariate models. This prevented undue influence from extreme FC observations. Observations used for this analysis were independent across patients but not within patients as we took multiple samples from individual patients. Robust variance estimates were used to account for the lack of independence of observations within patients to produce conservative estimates for $\mathrm{p}$ values. Stata 8.2 (College Station, Tex., USA) was used for the analyses. Reported $p$ values are two-tailed; $\mathrm{p}$ values $<0.05$ were considered significant.

\section{Results}

Fourteen VLBW infants were consecutively enrolled in this study from February to May 2006. Mean GA was $26.6 \pm 2.1$ weeks (range 23-30 weeks) and mean birth weight was $982.1 \pm 289.4 \mathrm{~g}$ (range $630-1,500 \mathrm{~g}$ ). There were 8 male, 6 female, 9 black and 5 white infants. Among the 14 infants, 2 were enrolled late, 4 were withdrawn early including 3 transferred to other hospitals and 1 death, and 8 completed the study. The maternal conditions included the 8 mothers with preterm laboring, 3 with prolonged rupture of membrane, 4 with preeclampsia, 1 with placental abruption, and 1 with hypertension 


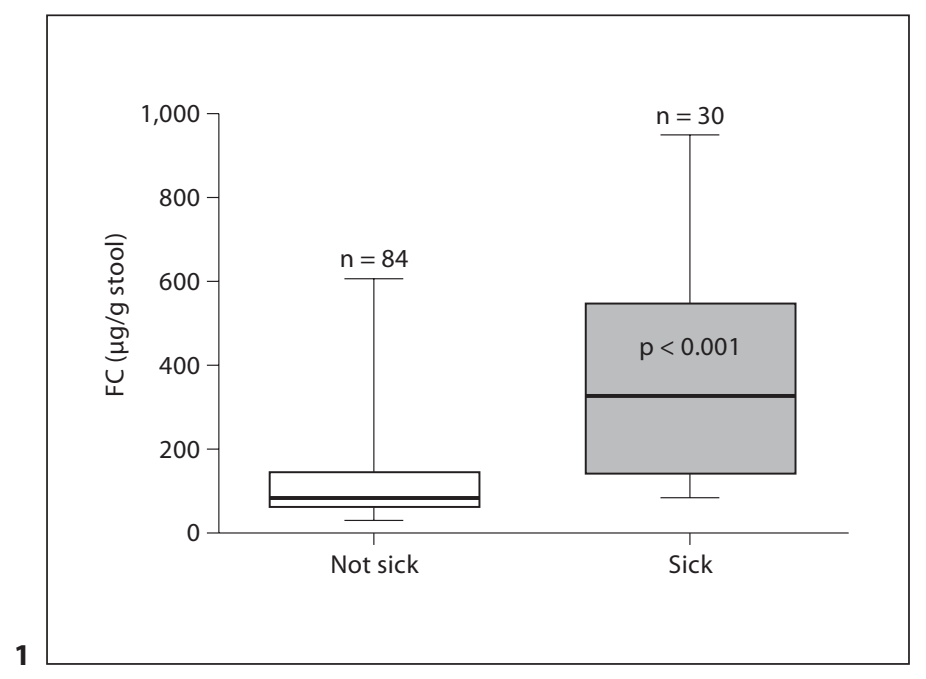

Fig. 1. FC level in infants characterized as 'not sick' versus 'sick'. Fig. 2. Dynamic change of FC level in response to minor systemic/gastrointestinal infection or stress and treatment (FC $<350 \mu \mathrm{g} /$ $\mathrm{g}$ stool) in specific infants. The spots pointed by arrows are when the infants developed clinical symptoms stated in corresponding boxes and treatments started. KUB $=$ Kidney ureter bladder; $\mathrm{PDA}=$ patent ductus arteriosus; UTI = urinary tract infection; $\mathrm{WBC}=$ white blood cells.

Fig. 3. Dynamic change of FC level in response to major systemic/ gastrointestinal infection or stress and treatment $(\mathrm{FC}>350 \mu \mathrm{g} / \mathrm{g}$ stool) in specific infants. The spots pointed by arrows are when the infants developed clinical symptoms stated in corresponding boxes and treatments started. UGI = Upper gastrointestinal series (for further abbreviations, see fig. 2).

and oligohydramnios. The delivery modes were 9 cesarean section and 5 spontaneous vaginal deliveries.

A total of 211 stool samples were collected, of which 114 were analyzed. Stool samples were selected for FC level determination to provide one sample each day for infants classified as 'sick' and one sample each day or every other day for infants classified as 'not sick', depending on the availability of stool samples collected. If an infant had multiple stool samples within $24 \mathrm{~h}$, the sample closer to the end of the 24-hour period was selected as the daily sample.

The mean FC level from the 114 stool samples was $190.6 \pm 189.1 \mu \mathrm{g} / \mathrm{g}$ stool (range 33.9-951.1 $\mu \mathrm{g} / \mathrm{g}$ stool). FC levels were not related to GA $(p=0.255)$ or feedings of maternal breast milk versus hydrolyzed formula ( $\mathrm{p}=$ 0.257). The FC level tended to decrease with increasing age $(\mathrm{p}=0.121)$ and feeding volumes $(\mathrm{p}=0.179)$, but these relationships were not statistically significant.
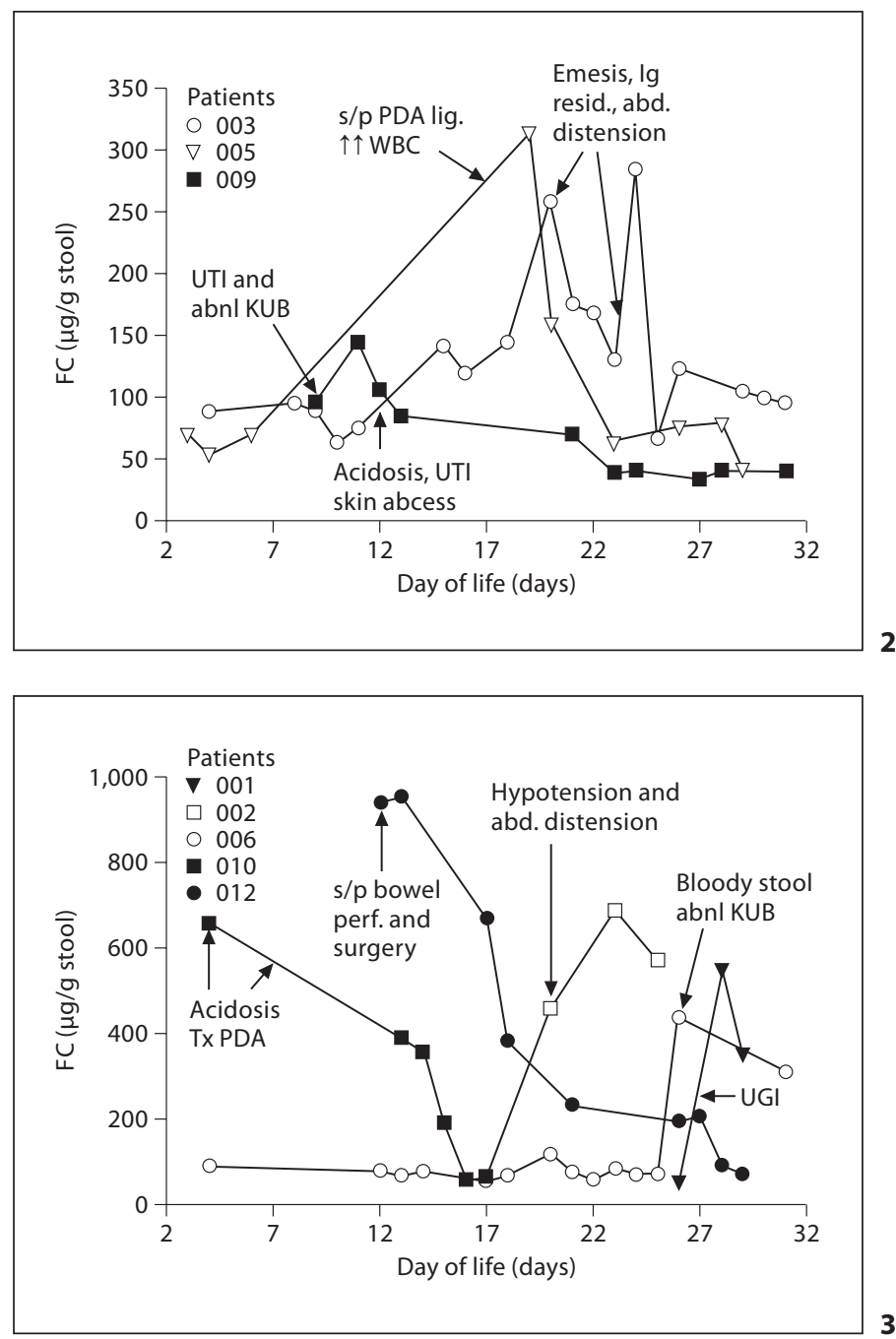

FC levels differed between infants classified as 'not sick' and as 'sick' (122.8 \pm 98.9 vs. $380.4 \pm 246.3 \mu \mathrm{g} / \mathrm{g}$ stool, $\mathrm{p}<0.001$ ) based on their clinical conditions (fig. 1). Increases in FC levels were observed in response to systemic/gastrointestinal infections or stress, and the magnitude of increase depended on the severity of infection or stress. Moderately increased FC $(<350 \mu \mathrm{g} / \mathrm{g}$ stool) was observed in 3 infants with minor infection or stress such as urinary tract infection, feeding intolerance and postpatent ductus arteriosus ligation (fig. 2), while greater elevations of FC $>350 \mu \mathrm{g} / \mathrm{g}$ stool were noted in 5 infants with major gastrointestinal manifestations such as bloody stool, bowel perforation, distended abdomen with hypotension and upper gastrointestinal contrast study (fig. 3). In all sick infants, FC levels decreased over time with treatments or recovery (fig. 2, 3). 


\section{Discussion}

The primary findings in this pilot study were that FC levels were not related to the GA at birth in VLBW infants and that in preterm infants FC levels rose around the time of initial signs of gastrointestinal illness and decreased as these signs were treated and resolved. Although no infants had NEC based on radiological findings, the FC levels were greater ( $>350 \mu \mathrm{g} / \mathrm{g}$ stool) among those with NEClike symptoms or direct gastrointestinal injury/stress (such as bloody stool, bowel perforation and surgery, gastrointestinal contrast study), or with other conditions (such as severe hypotension and acidosis) which could significantly affect the permeability of the gastrointestinal mucosa. Lower FC levels $(<350 \mu \mathrm{g} / \mathrm{g}$ stool) were seen in minor infection (such as urinary tract infection, skin abscess), feeding intolerance, or after minor surgery with elevated white blood cells. The elevated FC levels in all 8 infants dropped after treatments and/or recovery. FC levels appear related to systemic infection/inflammation or stress and recovery suggesting that FC is not a specific marker for diagnosing NEC. Our results suggest that FC levels could be used to indicate the severity of the gastrointestinal inflammation process and monitor the response to treatment. The rapidity of the decrease of FC levels during the recovery period of a gastrointestinal illness might be useful for deciding when to begin feeds and stop antibiotics.

As compared to term infants, the more immature of premature infants are, the more intestinal mucosa permeable is, which may lead to inflammation as neutrophils migrate into the gut lumen $[15,16]$. A gradual decrease in permeability may explain our observation that FC levels tended to decrease with increasing age.

Enteral feeding of even very small volumes promotes intestinal maturity in preterm infants [17-19] and can increase gastrointestinal motility and function [20, 21], promote lactose digestion and decrease intestinal permeability [18]. In agreement with others [17-19], we observed that FC levels tend to decrease with increasing enteral feeding volume during the first month of postnatal life. We found no difference in the FC levels of infants fed maternal breast milk versus hydrolyzed formula. Similarly, Campeotto et al. [10] did not detect an influence of the type of feedings on FC levels.

Biomarkers are most useful to clinicians when the sensitivity and specificity are high. Several groups have reported that term and preterm infants have a wide range and higher average FC levels $[8,10,11,13,14]$ than healthy adults ( $\approx 50 \mu \mathrm{g} / \mathrm{g}$ stool [22]). Similar to previous reports, we found a wide range and high average level of FC (190.6 $\pm 189.1 \mu \mathrm{g} / \mathrm{g}$ stool) from 114 stool samples of 14 VLBW infants. However, unlike the results from single stool samples, we serially measured FC levels daily or every other day to correlate the FC levels with infants' clinical status. After grouping the FC measurements according to the infants' clinical condition, FC levels in the samples from infants during their 'not sick' period were significantly lower than those from infants during their 'sick' period (fig. 1). When the FC level was $\leq 100 \mu \mathrm{g} / \mathrm{g}$ stool, the probability of an infant being sick was $2 \%$. The likelihood of an infant being sick increased from $32 \%$ when the FC level was between 101 and $300 \mu \mathrm{g} / \mathrm{g}$ stool to $74 \%$ when the FC level was $>300 \mu \mathrm{g} / \mathrm{g}$ stool.

It seems that FC might be a sensitive marker for gastrointestinal inflammatory conditions including NEC, as reported previously $[12,14]$. Josefsson et al. [12] found FC levels most dramatically elevated in VLBW infants diagnosed with NEC in comparison with those in reference VLBW infants with suspicion of NEC who had significantly increased FC levels as well. Carroll et al. [14] also found that the postdiagnosis FC level was significantly higher in infants with NEC compared to controls. However, the above studies did not provide any follow-up measurements of FC levels to monitor the response of NEC to treatment or examine the changes in FC levels for NEC versus other gastrointestinal manifestations.

We observed 4 VLBW infants who were clinically well, but had significantly elevated FC levels (300-600 $\mu \mathrm{g} / \mathrm{g}$ stool) in two meconium stool samples. This dropped to $\approx 100 \mu \mathrm{g} / \mathrm{g}$ stool by the end of the first week and continually decreased during the second week (data not shown). The mothers of the 4 infants presented conditions of prolonged rupture of membrane, cerclage placement with preeclampsia, and hypertension and oligohydramnios. Similarly, Josefsson et al. [12] reported a significant decrease in FC levels from week 0 (median $332 \mu \mathrm{g} / \mathrm{g}$ ) to week 1 (median $190 \mu \mathrm{g} / \mathrm{g}$ ) in VLBW infants. This suggested that maternal conditions may affect infants' FC levels after birth.

The major limitations of this pilot study are related to the small sample size, the limited observation period that may have not been long enough to cover the late-onset NEC occurring after the first month of life and lack of defined NEC cases. In addition, this pilot study limited the infants fed with only either maternal breast milk or hydrolysate formula which is not a common practice and thus our results might not apply to other formulas used for VLBW infants. 
In summary, VLBW infants had a wide range and higher than normal adult average level of FC. Higher FC levels were observed in infants with gastrointestinal injury or infection compared to other minor systemic infection or stress, and FC levels decreased as these illnesses resolved. Based on this pilot study with serial measurements of FC levels, we speculate that FC may be useful as an indicator for the presence and severity of NEC and the response to treatment. FC should be studied in a larger cohort of infants with an adequate incidence of NEC to assess its predictive value.

\section{Acknowledgements}

The authors specially thank all DUMC NICU nurses and fellows who helped to collect the stool samples, and Drs. Rick Auten (DUMC) and Paul Dawson (WFUBMC) for storing and transporting the samples. We also thank Dr. Michael O'Shea and Dr. Paul Dawson for critically reading the manuscript.

\section{References}

$\checkmark 1$ Henry MC, Moss RL: Current issues in the management of necrotizing enterocolitis. Semin Perinatol 2004;28:221-233.

$\checkmark 2$ Uauy RD, Fanaroff AA, Korones SB, Phillips EA, Phillips JB, Wright LL and the NICHD Neonatal Research Network: Necrotizing enterocolitis in very low birth weight infants: biodemographic and clinical correlates. $\mathrm{Na}$ tional Institute of Child Health and Human Development Neonatal Research Network. J Pediatr 1991;119:630-638.

$\checkmark 3$ Network TCN: Variations in incidence of necrotizing enterocolitis in Canadian neonatal intensive care units. J Pediatr Gastroenterol Nutr 2004;39:366-372.

4 Dale I, Brandtzaeg P, Fagerhol MK, Scott H: Distribution of a new myelomoncytic antigen (L1) in human peripheral blood leukocytes. Immunofluorescence and immunoperoxidase staining features in comparison with lysozyme and lactoferrin. Am J Clin Pathol 1985;84:24-34.

$\checkmark 5$ Roseth AG, Schmidt PN, Fagerhol MK: Correlation between faecal excretion of 111-indium labeled granulocytes and calprotectin, a granulocyte marker protein in patients with inflammatory bowel disease. Scand J Gastroenterol 1999;34:50-54.

6 Roseth AG, Aadland E, Jahnsen J, Raknerud $\mathrm{N}$ : Assessment of disease activity in ulcerative colitis by faecal calprotectin, a novel granulocyte marker protein. Digestion 1997; 58:176-180.
7 Carroccio A, Iacono G, Cottone M, Di Prima L, Cartabellotta F, Cavataio F, Scalici C, Montalto G, Di Fede G, Rini GB, Notarbartolo A, Averna MR: Diagnostic accuracy of fecal calprotectin assay in distinguishing organic causes of chronic diarrhea from irritable bowel syndrome: a prospective study in adults and children. Clin Chem 2003;49. 861-867.

8 Olafsdottir E, Aksnes L, Fluge G, Bersad A: Faecal calprotein levels in infants with infantile colic, healthy infants, children with inflammatory bowel disease, children with recurrent abdominal pain and healthy children. Acta Paediatr 2002;91:45-50.

-9 Rugtveit J, Fagerhol MK: Age-dependent variations in fecal calprotectin concentrations in children. J Pediatr Gastroenterol Nutr 2002;34:323-324.

10 Campeotto F, Butel MJ, Kalach N, Derrieux S, Aubert-Jacquin C, Barbot L, Francoual C, Dupont C, Kapel N: High faecal calprotectin concentrations in newborn infants. Arch Dis Child Fetal Neonatal Ed 2004;89:F353F355.

11 Nissen AC, van Gils CE, Menheere PP, Van den N, Anita M, van der Hoeven MA, Forget PP: Fecal calprotectin in healthy term and preterm infants. J Pediatr Gastroenterol Nutr 2004;38:107-108.

12 Josefsson S, Bunn SK, Domellöf M: Fecal calprotectin in very low birth weight infants. J Pediat Gastroenterol Nutr 2007;44:407-413.

13 Laforgia N, Baldassarre ME, Pontrelli G, Indrio F, Altomare MA, Di Bitonto G, Mautone A: Calprotectin levels in meconium. Acta Pediatr 2003;92:463-466.
14 Carroll D, Corfield A, Spicer R, Cairns P: Fecal calprotectin concentrations and diagnosis of necrotizing enterocolitis. Lancet 2003; 361:310-311.

15 Weaver LT, Laker MF, Nelson R: Intestinal permeability in the newborn. Arch Dis Child 1984;59:236-241.

16 Cummins AG, Thompson FM: Postnatal changes in mucosa immune response: a physiological perspective of breastfeeding and weaning. Immunol Cell Biol 1997;75: 419-429.

17 Berseth CL, Nordyke C: Enteral nutrients promote postnatal maturation of intestinal motor activity in preterm infants. Am J Physiol 1993;264:G1046-G1051.

18 Shulman RJ, Schanler RJ, Lau C, Heitkemper M, Ou CN, Smith EO: Early feeding, feeding tolerance, and lactase activity in preterm infants. J Pediatr 1998;133:645-649.

19 Sangild PT: Gut responses to enteral nutrition in preterm infants and animals. Exp Biol Med 2006;231:1695-1711.

20 Lucas A, Bloom SR, Aynsley-Green A: Gut hormones and 'minimal enteral feeding'. Acta Paediatr Scand 1986;75:719-723.

21 Berseth CL: Minimal enteral feedings. Clin Perinatal 1995;22:195-205.

22 Tøn H, Brandsnes Ø, Dale S, Holtlund J, Skuibina E, Schjønsby H, Johne B: Improved assay for fecal calprotectin. Clin Chim Acta 2000;292:41-54. 